\title{
Effect of sertraline in paroxysmal hypertension
}

\author{
Jan Vaclavika, Aneta Krenkovab ${ }^{b}$ Eva Kocianova ${ }^{a}$, Tomas Vaclavikc, Monika Kamasova ${ }^{a}$
}

\begin{abstract}
Objective. Paroxysmal hypertension or pseudopheochromocytoma is quite a common problem in clinical practice. The optimal treatment for this condition has not been established. This study sought to investigate whether sertraline (a selective serotonin reuptake inhibitor) reduces the symptoms.

Methods. We enrolled 64 patients referred to our department between April 2008 and October 2014 for symptomatic paroxysmal hypertension. Patients received sertraline, $50 \mathrm{mg}$ once daily, in addition to their current medication. The effect of the treatment was assessed during their next clinical visit at least 3 months later.

Results. Of the 64 patients, 57 (89\%) also had sustained arterial hypertension. Mean office baseline blood pressure (BP) was $147.6 / 83.8 \mathrm{mmHg}$ and patients used a mean of 3.1 antihypertensive drugs. Five patients did not start using sertraline and three were lost to follow-up. Of the 56 patients who started using sertraline and who came for check up, clinical improvement was observed in $42(75 \%)$ patients - symptoms of paroxysmal hypertension fully subsided in 28 $(50 \%)$ and were partially reduced in $14(25 \%)$. Side effects or intolerance leading to discontinuation of treatment occurred in 7 patients (12.5\%). Mean office BP in patients using sertraline decreased by $12.8 / 7.4 \mathrm{mmHg}(P<0.001$ for both). Conclusions. Sertraline effectively removed or reduced symptoms of paroxysmal hypertension in the majority of patients who used this treatment.
\end{abstract}

Key words: paroxysmal hypertension, pseudopheochromocytoma, treatment, selective serotonin reuptake inhibitors, sertraline

Received: June 28, 2017; Accepted: September 6, 2017; Available online: October 17, 2017 https://doi.org/10.5507/bp.2017.039

${ }^{a}$ Deparment of Internal Medicine I - Cardiology, University Hospital Olomouc and Faculty of Medicine and Dentistry, Palacky University Olomouc, Czech Republic

${ }^{b}$ Department of Pneumology, University Hospital Olomouc and Faculty of Medicine and Dentistry, Palacky University Olomouc, Czech Republic

'Statistics and Probability Department, Faculty of Informatics and Statistics, University of Economics in Prague, nam. W. Churchilla 1938/4, 13067 Prague, Czech Republic

Corresponding author: Jan Vaclavik, e-mail:vaclavik.j@centrum.cz

\section{INTRODUCTION}

Paroxysmal arterial hypertension, also called labile hypertension or pseudopheochromocytoma, is a quite common problem encountered by physicians in clinical practice. It is characterized by recurrent hypertensive episodes of abrupt onset, usually accompanied by symptoms of catecholamine excess, including palpitations, flushing, diaphoresis, anxiety and tremor ${ }^{1,2}$. Other symptoms might also be present, such as headache, chest pain, nausea or dizziness ${ }^{1,2}$.

Pseudopheochromocytoma is largely a diagnosis of exclusion, which requires careful screening for any treatable pheochromocytoma or other conditions before the diagnosis is made ${ }^{1,2}$. However, pheochromocytoma is found in less than $2 \%$ of patients tested for suspicion of this diagnosis ${ }^{3}$, and in large series it was found in only $0.33-0.72 \%$ of patients with paroxysmal hypertension ${ }^{4,5}$.

The pathophysiology of paroxysmal hypertension is not well understood, but most likely involves activation of the sympathetic nervous system ${ }^{6}$. The mechanism that underlies sympathetic activation is unclear but appears to involve emotional factors ${ }^{1}$. Psychosomatic causes seem to be important in the pathogenesis of this little explored entity. There is considerable overlap between pseudopheochromocytoma and panic disorder, but it is not known to what extent psychological factors can influence the acute rise in blood pressure seen in patients with pseudopheochromocytoma ${ }^{2}$.

Optimal treatment of both acute management and prevention of hypertensive paroxysms is not well defined. Treatment modalities for preventive management include antihypertensive drug therapy (usually combined $\alpha$ - and $\beta$-blockade), psychopharmacologic treatment and psychological interventions ${ }^{1}$. Antidepressant agents, including selective serotonin reuptake inhibitors (SSRI), were reported to prevent recurrence of paroxysms in most patients at dosages recommended for treating panic disor$\mathrm{der}^{7}$. However, evidence to guide treatment is very scarce and originating from limited sets of patients ${ }^{7}$.

To further elucidate the therapeutic role of antidepressant agents we decided to evaluate the effects of selective serotonin reuptake inhibitor sertraline in patients presenting with paroxysmal hypertension. 


\section{METHODS}

By a computerized search of an electronic hospital database we identified patients who were referred to Department of Internal Medicine I - Cardiology, University Hospital Olomouc between April 2008 and October 2014 and received a prescription of sertraline in an outpatient department. A manual search of hospital records subsequently selected those patients in whom sertraline was prescribed for symptomatic paroxysmal hypertension.

Hospital records of these patients were further searched for demographic data (age, sex), duration and frequency of hypertensive paroxysms prior presentation, maximum systolic and diastolic blood pressure (BP) during hypertensive paroxysms and physical symptoms accompanying paroxysmal hypertension (headache, nausea, sweating, flushes, vertigo, chest pain, dyspnea, palpitations or other symptoms). Baseline office systolic and diastolic BP, heart rate and laboratory values (serum sodium, potassium, glycaemia, creatinine, estimated glomerular filtration rate and albuminuria) were recorded, together with a number of antihypertensive drugs currently used. Significant comorbidities were recorded (history of permanent hypertension, diabetes mellitus, coronary artery disease, stroke or chronic kidney disease). Whenever available, mean 24-hour systolic and diastolic BP on ambulatory blood pressure monitoring was also recorded.

All patients received sertraline (a selective serotonin reuptake inhibitor, $50 \mathrm{mg}$ once daily) as an add-on to their

Table 1. Baseline characteristics of patients.

\begin{tabular}{lc}
\hline Characteristic & $\begin{array}{c}\text { Enrolled } \\
\text { patients } \\
(\mathrm{n}=64)\end{array}$ \\
\hline Age (years) & $67.1 \pm 11.9$ \\
Female sex $(\%)$ & $52(81.3 \%)$ \\
\hline Patient comorbidities & $57(89.1 \%)$ \\
Permanent hypertension & $14(20.3 \%)$ \\
Diabetes mellitus & $10(15.6 \%)$ \\
Coronary artery disease & $5(7.8 \%)$ \\
Stroke & $1(1.6 \%)$ \\
Chronic kidney disease & \\
\hline Blood pressure & $147.6( \pm 23.2)$ \\
Office systolic BP (mmHg) & $83.8( \pm 14.1)$ \\
Office diastolic BP $(\mathrm{mmHg})$ & $132.0( \pm 15.4)$ \\
24-hour ambulatory systolic BP $(\mathrm{mmHg})$ & $73.0( \pm 10.0)$ \\
24-hour ambulatory diastolic BP $(\mathrm{mmHg})$ & $70.1( \pm 14.9)$ \\
Office heart rate $\left(\mathrm{min}^{-1}\right)$ & $3.1( \pm 1.7)$ \\
Mean number of antihypertensive drugs used & \\
\hline Laboratory parameters & $140.7( \pm 3.0)$ \\
Serum sodium (mmol/L) & $4.2( \pm 0.4)$ \\
Serum potassium $(\mathrm{mmol} / \mathrm{L})$ & $6.4( \pm 1.9)$ \\
Serum glucose $(\mathrm{mmol} / \mathrm{L})$ & $80.7( \pm 14.9)$ \\
Serum creatinine $(\mu \mathrm{mol} / \mathrm{L}$ & $1.1( \pm 0.3)$ \\
eGFR (mL/s/1.73 m $\left.{ }^{2}\right)$ &
\end{tabular}

$\mathrm{BP}=$ blood pressure; eGFR = estimated glomerular filtration rate (calculated according to the MDRD formula) current medication. Effect of this treatment was assessed at the next clinical visit at least 3 months later as either complete remission and/or partial regression of hypertensive paroxysms. Office BP, heart rate and number of antihypertensive drugs at follow up were also recorded. Results of biochemical and imaging testing for pheochromocytoma were reviewed. If a patient was not using sertraline on next clinical visit, reasons for its withdrawal were identified in the hospital records.

Standard descriptive statistics were applied to the recorded data. Results are presented as numbers (\%) or mean \pm standard deviation. A paired t-test was used for comparison of patient blood pressure changes at baseline and end of the follow up.

\section{RESULTS}

Between April 2008 and October 2014, a total of 64 patients referred to our department for symptomatic paroxysmal hypertension were prescribed sertraline, $50 \mathrm{mg}$ orally once daily.

Baseline characteristics of the patients are shown in Table 1. Most enrolled patients were predominantly older women $(81.3 \%$, mean age 67 years), of whom the majority $(89.1 \%)$ also had a permanent (chronic) arterial hypertension and were treated by a mean of 3.1 antihypertensive drugs. Mean baseline office BP was 147.6/83.8 mmHg, 27 patients $(42.2 \%)$ were normotensive at baseline with office both systolic BP $<140 \mathrm{mmHg}$ and diastolic BP $<90$ $\mathrm{mmHg}$. Only a small proportion of patients had a history of a manifest cardiovascular disease prior to enrollment.

A detailed characteristics of hypertensive paroxysms and accompanying symptoms are shown in Table 2. Mean BP during the paroxysm was $188.1 / 102.5 \mathrm{mmHg}$ and this represented a mean $40 \mathrm{mmHg}(30.1 \%)$ increase compared to their resting office systolic BP. Most patients had hypertensive paroxysms on a weekly basis and mean time from symptom onset to referral was 6.2 months. With the exception of 4 patients, who only had sole elevation of BP, most patients experienced various accompanying symptoms during hypertensive paroxysms, of which most common were anxiety, palpitations, headache and chest pain (please see Table 2).

17 patients $(26.6 \%)$ were not using sertraline on the next clinical visit at least 3 months later: 5 did not start using sertraline, 3 were lost to follow-up and 9 withdrew spontaneously before the next visit ( 3 for gastrointestinal side effects, 4 for other unspecified intolerance, 1 for spontaneous symptom cessations and 1 for lack of clinical effect).

Of the remaining 47 patients who were using sertraline on the next clinical visit, 42 (89.4\%) reported clinical improvement after this treatment. In this "on-treatment" analysis, symptoms of paroxysmal hypertension fully subsided in $28(59.6 \%)$ and were partially reduced in $14(29.8 \%)$. Mean office BP in patients using sertraline decreased by $12.8 / 7.4 \mathrm{mmHg}(P<0.001$ for both). At the final visit, mean office BP was $133.4( \pm 20.8) / 75.9$ 
$( \pm 12.1) \mathrm{mmHg}$, patients still used a mean of $3.1( \pm 1.3)$ antihypertensive drugs. Mean heart rate at the final visit was $68.0( \pm 10.2) \mathrm{min}^{-1}$.

In the "intention-to-treat" analysis of all 56 patients who started using sertraline and presented at the next clinical visit, clinical improvement was observed in 42 (75\%) patients - symptoms of paroxysmal hypertension fully subsided in 28 (50\%) and were partially reduced in $14(25 \%)$ patients. Side effects or intolerance leading to discontinuation of treatment occurred in 7 patients (12.5\%) (Please see Fig. 1).

A diagnosis of pheochromocytoma was not established in any of the 64 patients, based on biochemical testing, imaging methods and long-term follow up. Free plasma metanephrines were analyzed in the majority of patients (86\%). Isolated elevation of plasma metanephrine in the grey zone ${ }^{8}$ was found in 8 patients and isolated elevation of plasma normetanephrine in the grey zone in 5 patients, but all these patients had negative adjustment of plasma metanephrines for age ${ }^{9}$ and negative results of imaging for pheochromocytoma or paraganglioma. Elevation of both plasma metanephrine and normetanephrine in the grey zone was present in 2 patients, of whom one 87-year old woman was lost to follow up and one 55-year old woman had negative results of multiple imaging methods.

\section{DISCUSSION}

This study showed that sertraline might be a very effective therapeutic measure in the chronic management of paroxysmal hypertension (pseudopheochromocytoma). After the period of at least three months of treatment, sertraline led to significant clinical improvement in $75 \%$ patients and complete regression of symptoms in half of the patients. Among those who tolerated the treatment well, the success rates were even higher.

To our knowledge, this is the largest therapeutic study in the field of paroxysmal hypertension reported to this date. One of the largest previously published series included 21 patients, of whom successful outcome of treatment was achieved in 13 (62\%) and majority of them required prescription of an antidepressant ${ }^{7}$.

Our study points to the major importance of emotional and psychosomatic causes in the pathogenesis of paroxysmal hypertension, which, as our results show, can be positively influenced by selective serotonin reuptake inhibitors (SSRI). Although paroxysmal hypertension, in contrast to panic disorder, is not anteceded or triggered by fear or panic ${ }^{7}$, these two units are sometimes difficult to distinguish in clinical practice ${ }^{10}$. The clinical picture of panic disorder to certain extent overlaps with pseudopheochromocytoma and both may well represent different manifestations of the same disorder entity ${ }^{11}$.

The biochemical basis for panic disorder may be an increased brain serotonin turnover, which was found to be 4 times higher in patients with panic disorder than in normal controls ${ }^{12}$. These findings may explain why SSRI are very effective in managing panic disorder. Given very similar clinical symptoms of panic disorder and pseu-
Table 2. Characteristics of hypertensive paroxysms.

\begin{tabular}{|c|c|}
\hline Characteristic & $\begin{array}{l}\text { Enrolled } \\
\text { patients } \\
(\mathrm{n}=64)\end{array}$ \\
\hline Maximum systolic BP (mmHg) & $188.1( \pm 24.9)$ \\
\hline Maximum diastolic BP (mmHg) & $102.5( \pm 12.6)$ \\
\hline $\begin{array}{l}\text { Mean duration from the onset of symptoms } \\
\text { (months) }\end{array}$ & $6.2( \pm 14.4)$ \\
\hline \multicolumn{2}{|l|}{ Frequency of symptoms } \\
\hline Daily & $11(17.2 \%)$ \\
\hline Weekly & $30(46.9 \%)$ \\
\hline Monthly & $9(14.1 \%)$ \\
\hline Not reported & $14(21.9 \%)$ \\
\hline \multicolumn{2}{|l|}{ Degree of BP elevation } \\
\hline Maximum systolic $\mathrm{BP} \geq 200 \mathrm{mmHg}$ & $27(42.2 \%)$ \\
\hline Maximum systolic $\mathrm{BP} \geq 180 \mathrm{mmHg}$ & $10(15.6 \%)$ \\
\hline Maximum systolic $\mathrm{BP} \geq 160 \mathrm{mmHg}$ & $16(25.0 \%)$ \\
\hline $\begin{array}{l}\text { Mean difference between systolic BP } \\
\text { during paroxysm and office systolic BP } \\
(\mathrm{mmHg})\end{array}$ & $40.0( \pm 33.9)$ \\
\hline $\begin{array}{l}\text { Mean difference between systolic BP } \\
\text { during paroxysm and office systolic BP (\%) }\end{array}$ & $30.1 \%( \pm 28.3 \%)$ \\
\hline \multicolumn{2}{|c|}{ Clinical symptoms accompanying hypertensive paroxysm } \\
\hline Nausea & $13(20.3 \%)$ \\
\hline Headache & $18(28.1 \%)$ \\
\hline Sweating & $4(6.3 \%)$ \\
\hline Vertigo & $11(17.2 \%)$ \\
\hline Flush & $10(15.6 \%)$ \\
\hline Chest pain & $17(26.6 \%)$ \\
\hline Dyspnoea & $13(20.3 \%)$ \\
\hline Palpitations & $19(29.7 \%)$ \\
\hline Depressive feelings & $8(12.5 \%)$ \\
\hline Anxiety & $22(34.4 \%)$ \\
\hline Nervousness & $7(10.9 \%)$ \\
\hline Ear humming & $6(9.4 \%)$ \\
\hline Skin paraesthesia & $4(6.3 \%)$ \\
\hline Inner tremor & $4(6.3 \%)$ \\
\hline Weakness & $2(3.1 \%)$ \\
\hline Fatigue & $3(4.7 \%)$ \\
\hline Pallor & $1(1.6 \%)$ \\
\hline Visual disturbances & $1(1.6 \%)$ \\
\hline Hot feelings & $2(3.1 \%)$ \\
\hline Burning sensations & $2(3.1 \%)$ \\
\hline Chills & $4(6.3 \%)$ \\
\hline $\begin{array}{l}\text { Only BP elevation, without any } \\
\text { accompanying symptoms }\end{array}$ & $4(6.3 \%)$ \\
\hline
\end{tabular}

$\mathrm{BP}=$ blood pressure

dopheochromocytoma ${ }^{11}$, it is possible that increased brain serotonin turnover also plays an important role in the pathogenesis of pseudopheochromocytoma, and this may explain very good therapeutic effect of SSRI in our patients.

In another study, patients with pseudopheochromocytoma had, in comparison with controls, documented comparable normal values of serum noradrenaline and normetanephrine, but increased serum adrenaline (by $120 \%$ ) and metanephrine (by 80\%) ( ref. $^{6}$ ), which suggested an increased adrenomedullary activation in this entity. In our study, we observed a mild elevation of either plasma metanephrine or normetanephrine in the grey 


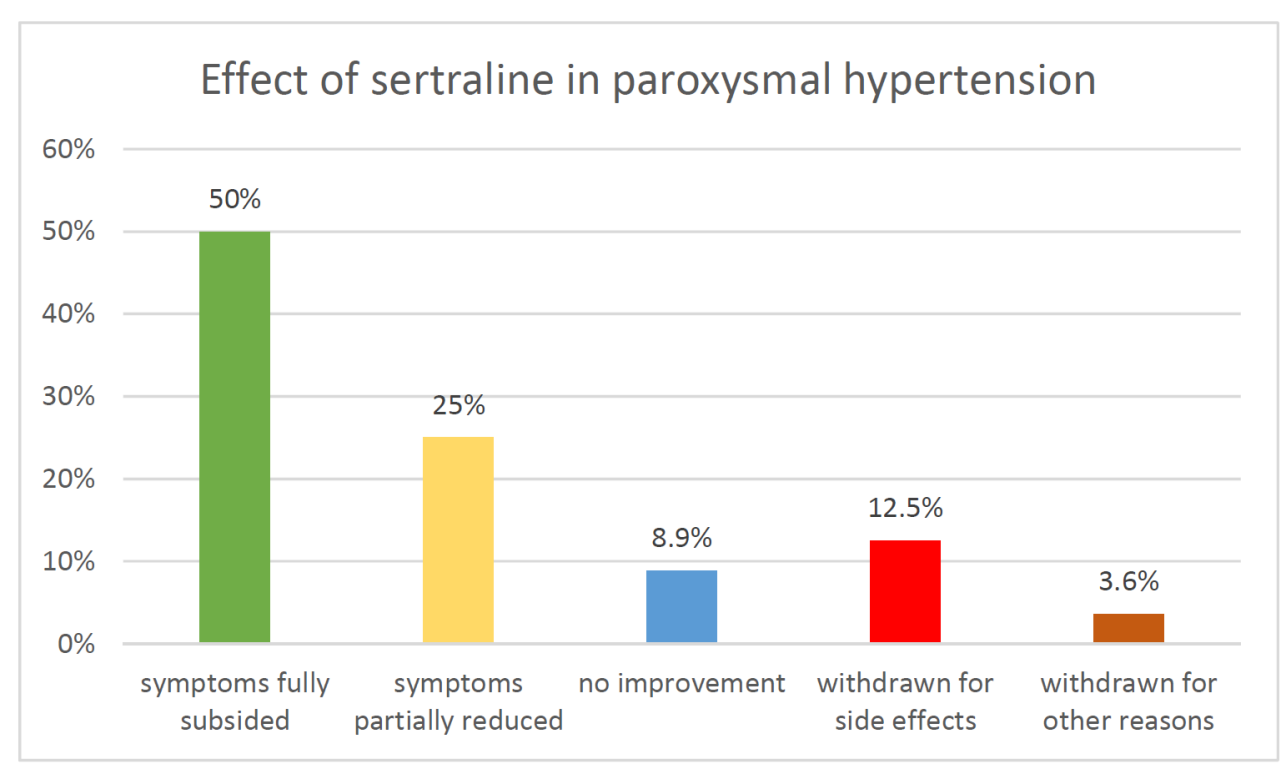

Fig. 1. Effect of sertraline in paroxysmal hypertension.

Symptoms of paroxysmal hypertension fully subsided in 50\% and were partially reduced in $25 \%$ patients after 3 months of treatment with sertraline. In $12.5 \%$ patients, administration of sertraline had to be stopped for side effects.

zone in $27 \%$ of the patients (elevation of both metanephrines only in $3.6 \%$ ). This rate is comparable to $29 \%$ of false positive results found in other studies when testing for pheochromocytoma because of suggestive symptoms while performing blood sampling in seated non-fasting patients as we $\mathrm{did}^{13}$. However, since the information on normal metanephrine values and occurrence of false positive results in healthy adults and other populations without suggestive symptoms is very limited, it is now impossible to judge the potential sympathetic or adrenal hyperactivity in our patients based on these laboratory values. Plasma adrenaline and noradrenaline analyses are no more routinely performed at our institution or elsewhere when excluding pheochromocytoma ${ }^{14}$.

There is no existing exact definition of paroxysmal hypertension ${ }^{11}$, which made the selection of patients for the study difficult. Our patients presented usually with severe elevations of blood pressure during paroxysms, which was on average by $40 \mathrm{mmHg}$ higher (or, relatively, by $30 \%$ higher) than their office BP. Although the majority of patients experienced one or more other symptoms accompanying hypertensive paroxysms, these symptoms were not present in all patients, so their presence is probably not required for future definition or diagnosis of paroxysmal hypertension.

In the light of our study, we would suggest defining paroxysmal hypertension clinically as abrupt elevations of resting systolic $\mathrm{BP} \geq 20 \%$ compared to previous measured systolic BP value before paroxysm, or $\geq 20 \%$ compared to mean systolic BP on 24-hour ambulatory blood pressure monitoring (ABPM), or $\geq 20 \%$ compared to measured office systolic BP, documented by a clinician or home blood pressure monitor, which require physician or emergency room visit or the use of any rescue antihypertensive medication by the patient.
Besides treatment of depression, SSRI are also approved for treatment of other conditions, including panic disorder. Sertraline was chosen out of the SSRI group and prescribed to our patients at our department based on the results of a meta-analysis, which showed that escitalopram and sertraline were the only two drugs among SSRI in both the most efficacious and best-tolerated groups ${ }^{15}$. Of the two, sertraline might be the better choice for initial treatment because of its lower cost at that time ${ }^{15}$. A dose of $50 \mathrm{mg}$ sertraline once daily, as in our study, is a commonly recommended initial dose for depression ${ }^{16}$. However, initiating the treatment with $25 \mathrm{mg}$ once daily for the first week could reduce the frequency of early treatment emergent side effects ${ }^{16}$, which led in our study to premature discontinuation of sertraline in $12.5 \%$ patients.

A limitation of our study is its retrospective and uncontrolled character. Several biases may have influenced the results. However, given the common occurrence of pseudopheochromocytoma in clinical practice and very limited therapeutic evidence, we feel that the clinicians should be informed about the efficacy of this treatment.

\section{CONCLUSION}

In conclusion, sertraline seems to be an effective treatment for patients with paroxysmal hypertension, leading to significant clinical improvement in $75 \%$ and complete regression of symptoms in half of the patients. Further research of this little explored entity is fully warranted. A prospective, randomized, placebo controlled trial of sertraline in paroxysmal hypertension would be timely, and is already being organized (ClinicalTrials.gov, number NCT02641652). 
Author contributions: JV: initiated the research and drafted the manuscript; JV, AK, MK, EK: performed data collection; TV: performed statistical analysis; all the authors critically revised the abstract and approved the paper prior to submission.

Conflict of Interest: None declared.

Presented at: $25^{\text {th }}$ European Meeting on Hypertension and Cardiovascular Prevention, Milan, June 12-15, 2015

\section{REFERENCES}

1. Mann SJ. Severe paroxysmal hypertension (pseudopheochromocytoma). Curr Hypertens Rep 2008;10(1):12-8.

2. Mackenzie IS, Brown MJ. Pseudopheochromocytoma. J Hypertens 2007;25(11):2204-6.

3. Václavík J, Stejskal D, Lačňák B, Lazárová M, Jedelský L, Kadalová $L$, Janosová $M$, Fryšák Z, Vlček P. Free plasma metanephrines as a screening test for pheochromocytoma in low-risk patients. J Hypertens 2007;25(7):1427-31.

4. Kuchel O. New Insights Into Pseudopheochromocytoma and Emotionally Provoked Hypertension. In: Clinical Hypertension and Vascular Diseases. Humana Press 2004:251-76.

5. Fogarty J, Engel C, Russo J. Hypertension and pheochromocytoma testing: the association with anxiety disorders. Arch Fam Med 1994;3:55-60.

6. Sharabi Y, Goldstein DS, Bentho O, Saleem A, Pechnik S, Geraci MF, Holmes C, Pacak K, Eisenhofer G. Sympathoadrenal function in patients with paroxysmal hypertension: pseudopheochromocytoma. Hypertens 2007;25(11):2286-95.

7. Mann SJ. Severe paroxysmal hypertension (pseudopheochromocytoma): understanding the cause and treatment. Arch Intern Med 1999;159(7):670-4.
8. Eisenhofer G, Goldstein DS, Walther MM, Friberg P, Lenders JW, Keiser HR, Pacak K. Biochemical diagnosis of pheochromocytoma: how to distinguish true- from false-positive test results. J Clin Endocrinol Metab 2003:88(6):2656-66.

9. Sawka AM, Thabane L, Gafni A, Levine M, Young WF. Measurement of fractionated plasma metanephrines for exclusion of pheochromocytoma: Can specificity be improved by adjustment for age? BMC Endocr Disord 2005;5(1):1.

10. Eisenhofer G, Sharabi Y, Pacak K. Unexplained symptomatic paroxysmal hypertension in pseudopheochromocytoma: A stress response disorder? Ann N Y Acad Sci 2008;1148:469-78.

11. Pickering TG, Clemow L. Paroxysmal hypertension: the role of stress and psychological factors. J Clin Hypertens (Greenwich) 2008;10(7):575-81.

12. Esler M, Lambert E, Alvarenga M, Socratous F, Richards J, Barton D, Pier C, Brenchley C, Dawood T, Hastings J, Guo L, Haikerwal D, Kaye D, Jennings G, Kalff V, Kelly M, Wiesner G, Lambert G. Increased brain serotonin turnover in panic disorder patients in the absence of a panic attack: reduction by a selective serotonin reuptake inhibitor. Stress 2007;10(3):295-304.

13. Därr R, Pamporaki $C$, Peitzsch $M$, Miehle K, Prejbisz A, Peczkowska M, Weismann D, Beuschlein F, Sinnott R, Bornstein SR, Neumann HP, Januszewicz A, Lenders J, Eisenhofer G. Biochemical diagnosis of phaeochromocytoma using plasma-free normetanephrine, metanephrine and methoxytyramine: importance of supine sampling under fasting conditions. Clin Endocrinol (Oxf) 2014;80(4):478-86.

14. Lenders JWM, Duh Q-Y, Eisenhofer G, Gimenez-Roqueplo AP, Grebe SK, Murad MH, Naruse M, Pacak K, Young WF Jr; Endocrine Society. Pheochromocytoma and paraganglioma: an endocrine society clinical practice guideline. J Clin Endocrinol Metab 2014;99(6):1915-42.

15. Cipriani A, Furukawa TA, Salanti G, Geddes JR, Higgins JP, Churchill R, Watanabe N, Nakagawa A, Omori IM, McGuire H, Tansella M, Barbui C. Comparative efficacy and acceptability of 12 new-generation antidepressants: a multiple-treatments meta-analysis. Lancet 2009;373(9665):746-58.

16. Zoloft - SUMMARY OF PRODUCT CHARACTERISTICS.; 2009. http:// www.ema.europa.eu/docs/en_GB/document_library/Referrals_document/Zoloft_30/WC500007023.pdf. 